EPJ manuscript No.

(will be inserted by the editor)

\title{
Controllable 3D atomic Brownian motor in optical lattices
}

\author{
Claude M. Dion ${ }^{1, a}$, Peder Sjölund ${ }^{1}$, Stefan J. H. Petra ${ }^{1}$, Svante Jonsell ${ }^{1, b}$, Mats Nylén ${ }^{1}$, \\ Laurent Sanchez-Palencia ${ }^{2}$, and Anders Kastberg ${ }^{1}$ \\ 1 Department of Physics, Umeå University, SE-90187 Umeå, Sweden \\ 2 Laboratoire Charles Fabry de l'Institut d'Optique, CNRS, Univ. Paris-Sud, Campus Polytechnique, \\ RD-128, F-91127 Palaiseau cedex, France
}

\begin{abstract}
We study a Brownian motor, based on cold atoms in optical lattices, where atomic motion can be induced in a controlled manner in an arbitrary direction, by rectification of isotropic random fluctuations. In contrast with ratchet mechanisms, our Brownian motor operates in a potential that is spatially and temporally symmetric, in apparent contradiction to the Curie principle. Simulations, based on the Fokker-Planck equation, allow us to gain knowledge on the qualitative behaviour of our Brownian motor. Studies of Brownian motors, and in particular ones with unique control properties, are of fundamental interest because of the role they play in protein motors and their potential applications in nanotechnology. In particular, our system opens the way to the study of quantum Brownian motors.
\end{abstract}

\section{Introduction}

Brownian motors are devices capable of converting the energy of the random, isotropic motion of Brownian particles into useful work, for instance driving the particles into a directed motion, without any macroscopic force [1,2]. This possibility is not trivial in the face of fundamental symmetry and thermodynamic laws. Indeed, realising a Brownian motor requires that the system must be (i) asymmetric and (ii) brought out of thermodynamic equilibrium. On one hand, the need for asymmetry to extract directed motion out of random fluctuations is intuitive and is underpinned by the Curie principle, which states that asymmetric dynamics cannot emerge in a system possessing both spatial and temporal symmetries 3 . On the other hand, the need for working out of equilibrium comes from the second law of thermodynamics, which states that the total entropy always increases. Surprisingly enough, these two requirements are generally sufficient for realising a Brownian motor, although no rigorous proof is available so far 2]. In his lectures of physics, Richard Feynman describes a seminal ratchet mechanism able to rectify noise [4, based on an original idea of Smoluchowski [5]. Up to now, essentially all suggestions and tentative demonstrations of ratchet effects and Brownian motors rely on that archetype principle, based on the application of a force, asymmetric either in space or in time, albeit one whose macroscopic average vanishes. However, it was suggested in ref. [6] that a ratchet effect can be induced in spatially and temporally symmetric potentials, provided that asymmetric jumps occur between potentials that are spatially shifted. It should be noted also that previous realisations of Brownian motors typically inherently lack the possibility

\footnotetext{
${ }^{a}$ e-mail: claude.dion@tp.umu.se

b Current address: Department of Physics, University of Wales Swansea, Singleton Park, Swansea SA2 8PP, United Kingdom
} 
of inducing motion in any direction in three dimensions and are difficult to control, as the asymmetry is built into the system.

Interestingly, systems of cold atoms in dissipative optical lattices offer plenty of possibilities to investigate standard problems of statistical physics with an unprecedented accuracy [7]. Indeed, these have proved to be highly controllable and versatile systems [7.8. Hence, dissipative optical lattices have been used recently to study several effects, such as mechanical bistability [9, 10, spatial diffusion in random or quasi-periodic structures [11,12,13, 14, and stochastic resonance [15, 16, 17. Ratchet effects have also been investigated in dissipative optical lattices with either a spatial [18, 19, or a temporal asymmetry [20, 21, 22, 23.

In this work, we review our recent experimental realisation of a Brownian motor based on the model proposed in ref. [6], using an ultra-cold gas of atoms trapped in a stationary dissipative double optical lattice [24], and provide further analysis of the rectification mechanism. Our Brownian motor operates with a mechanism where the potentials are both temporally and spatially symmetric. Moreover, in contrast to other rectification mechanisms reported so far, the motion can be induced in any direction in three dimensions with a controlled speed. This new type of Brownian motor opens up possibilities for fundamental studies of noise-induced directed motion. The underlying principle is very general and is potentially transferable to molecular motors and to applications in nano-electronics and chemistry [6].

We start by presenting in sec. 2 the basic working principle of our Brownian motor. Then, in sec. 3. we discuss its experimental realisation with cold atoms in a double optical lattice. In sec. 4 we investigate the qualitative behaviour of the Brownian motor, using classical simulations based on the Fokker-Planck equation. Then, we discuss in sec. 5 how this system could be extended to a regime relevant for the study of quantum Brownian motors. Finally, we summarise our results in sec. 6 .

\section{Brownian motor with symmetric potentials}

The basic rectification mechanism demonstrated in this work is depicted in fig. 1 (see also refs. 6, 24]). Depending on their internal state, the atoms are subjected to one of two three-dimensional periodic potentials $\left(U_{\mathrm{A}}\right.$ and $U_{\mathrm{B}}$, represented in 1D in fig. 1) with identical periods. In the simplest situation, the potentials are identical but possibly spatially shifted. At sufficiently low temperature, the atoms are trapped in the wells of the potentials. In addition, they are assumed to undergo a Brownian motion in each potential and can be transferred to a neighbouring site via thermal activation. Ever so often, an atom will be pumped from one internal state to the other, resulting in random jumps between potentials $U_{\mathrm{A}}$ and $U_{\mathrm{B}}$, with rates $\gamma_{\mathrm{A} \rightarrow \mathrm{B}}$ and $\gamma_{\mathrm{B} \rightarrow \mathrm{A}}$.

(a)

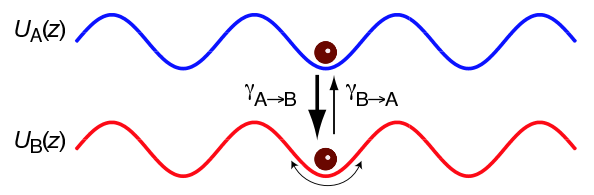

(b)

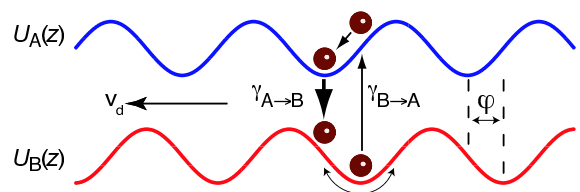

Fig. 1. Rectification mechanism. Atoms move in two symmetric potentials $U_{\mathrm{A}}(z)$ and $U_{\mathrm{B}}(z)$ that are coupled via the asymmetric optical pumping rates $\gamma_{A \rightarrow B}$ and $\gamma_{B \rightarrow A}\left(\gamma_{A \rightarrow B} \gg \gamma_{B} \rightarrow A\right)$. a) The potentials are in phase. The transfer from the long lived state B, to the transient state A, and back, will not lead to biased motion. b) A phase shift $\varphi(\neq 0, \pi)$ is introduced. Spatial diffusion will be strongly facilitated in one direction, leading to a drift velocity $v_{\mathrm{d}}$. [Taken from P. Sjölund, S. J. H. Petra, C. M. Dion, S. Jonsell, M. Nylén, L. Sanchez-Palencia, and A. Kastberg, Phys. Rev. Lett. 96, 190602 (2006). Copyright (2006) by the American Physical Society.] 
The asymmetry that eventually gives rise to controlled rectification is caused by a pronounced difference in the transfer rates between the potentials $\left(\gamma_{A \rightarrow B} \neq \gamma_{B \rightarrow A}\right)$. In the case where $\gamma_{\mathrm{A} \rightarrow \mathrm{B}}>\gamma_{\mathrm{B} \rightarrow \mathrm{A}}$, an atom will spend most of the time in the long lived state (B), and will oscillate near the bottom of a trapping site. At random times, it is pumped to the transient state (A), from where it returns quickly to state B. This excursion may drastically affect the motion of the atoms.

If the two potentials are identical and in phase [fig. 1(a)], this excursion will not affect the dynamics of the atoms. If the potentials are different, but still in phase, the change of state may result in a slight heating and increase the probability for an atom to be transferred to a neighbouring trapping site. This diffusion is symmetric. Qualitatively, the same occurs if the potentials are shifted by half a spatial period of the lattices, since no direction is favoured. The situation changes drastically if the relative phase between the potentials is shifted [fig. [1(b)]. During the time spent in lattice A, the atom experiences a potential with an incline that depends on the phase shift. The diffusion is then enhanced in a given direction, and correspondingly reduced in the opposite direction. While the potentials are symmetric and stationary, the atoms are propelled in a specific direction, which can be controlled by modifying the phase shift.

It is straightforward to extend the principle to three-dimensional potentials and to obtain a controlled motion in any direction. This mechanism works despite the absence of spatial as well as of temporal asymmetry, in apparent contradiction to the Curie principle 3. Instead, the combined dynamics, made up of phases of Hamiltonian motion interrupted by stochastic dissipative processes, provides the asymmetry [6. In our system, the directed motion is induced for atoms switching between two state-dependent periodic potentials that are coupled via optical pumping. The rectification process emanates from the fact that the couplings between the two potentials used, via the vacuum field reservoir, are strongly asymmetric.

\section{Experimental realisation}

This Brownian motor is realised, in a completely controllable fashion, using cold caesium atoms in a double optical lattice [24,30,31,32. The interaction of an atom with the interference pattern from a number of laser beams creates a spatially periodic potential (optical lattice), due to a second-order interaction between an atomic dipole and the light field [7,8. With the laser frequency of the optical lattice close to an atomic resonance, spontaneous emission of photons leads to dissipation. In addition to the trapping potential, we then have Sisyphus cooling [25], 26 27, providing friction and momentum diffusion in our system, and resulting in a Brownian motion of the atoms 28,29 .

In our setup, described in detail in refs. 30,31,32, we superpose two optical lattices, each being formed from the interference of four laser beams, resulting in tetragonal lattice structures [7,33. In lattice A, the atomic state trapped is the $F_{\mathrm{g}}=3$ hyperfine structure levels of the ground state of Cs $\left(6 \mathrm{~s}^{2} \mathrm{~S}_{1 / 2}\right)$, while lattice $\mathrm{B}$, spatially overlapped with the former, traps atoms in the $F_{\mathrm{g}}=4$ state. The difference in energy between these two ground states is large enough to enable spectrally selective optical lattices, while at the same time being small enough that the difference in the periodicity of the lattices (which depends on the wavelength of the light) is negligible on the physical scale of the region of interaction with the atoms.

The lasers are tuned near the D2 resonance $\left(6 \mathrm{~s}^{2} \mathrm{~S}_{1 / 2} \rightarrow 6 \mathrm{p}{ }^{2} \mathrm{P}_{3 / 2}\right)$ at $852 \mathrm{~nm}$. More specifically, lattices $\mathrm{A}$ and $\mathrm{B}$ are tuned close to the $F_{\mathrm{g}}=3 \rightarrow F_{\mathrm{e}}=4$ and $F_{\mathrm{g}}=4 \rightarrow F_{\mathrm{e}}=5$ resonances, respectively. The latter is a closed transition, so the rate of optical pumping out of lattice B will be slow, while the former is an open transition, such that the probability of optical pumping from lattice A to lattice B is high. The relative spatial phase between the two lattices can be controlled accurately, along any direction in space, by changing the path lengths of the individual laser beams $30,31,32$.

The experimental sequence is as follows. We start with a cloud of laser-cooled Cs atoms at a temperature of a few microkelvins. The two optical lattices are then turned on, trapping some $10^{8}$ atoms, with a filling fraction of about $5 \%$. The atoms are left to interact with the lattices for a time $\tau$, after which the lattice beams are abruptly turned off. Since no trapping potential 


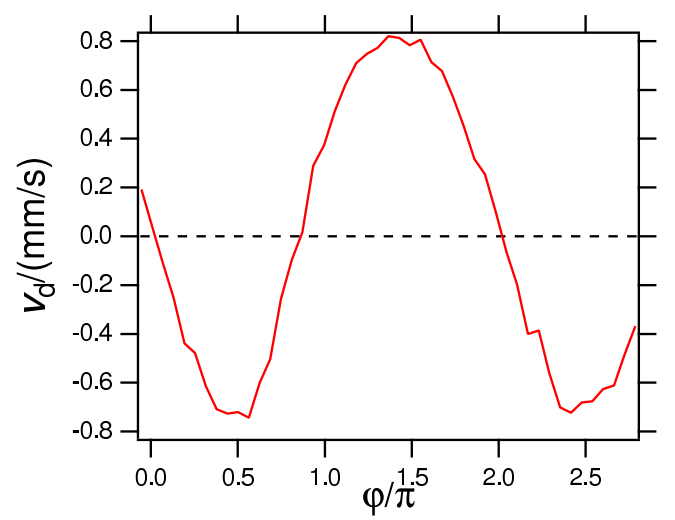

Fig. 2. Induced directed drift in the vertical $(z)$ direction as a function of the relative spatial phase $\varphi$ for an interaction time $\tau$ of $350 \mathrm{~ms}$.

is present any longer, the atoms will fall due to gravity and reach a detection probe located $\sim 5 \mathrm{~cm}$ below the interaction region with the optical lattices. This time-of-flight technique 27. gives the arrival time of atoms at the probe, which will depend on both the vertical $(z)$ position and the velocity of the atoms at the time of release. The initial velocity of the atoms in the double optical lattice can then be straightforwardly extracted from this arrival time, provided the distance between the interaction region and the probe is known 24 .

Sample data are presented in fig. 2. where the velocity of the atomic cloud in the double optical lattice is plotted as a function of the spatial shift between the lattices, for lattice A detuned $35.9 \Gamma$ from the $F_{\mathrm{g}}=3 \rightarrow F_{\mathrm{e}}=4$ transition with an irradiance of $0.5 \mathrm{~mW} / \mathrm{cm}^{2}$ per laser beam, and lattice $\mathrm{B}$ detuned $40.0 \Gamma$ from the $F_{\mathrm{g}}=4 \rightarrow F_{\mathrm{e}}=5$ transition, with $6.1 \mathrm{~mW} / \mathrm{cm}^{2}$ per beam ( $\Gamma$ is the natural linewidth of the $6 \mathrm{p}^{2} \mathrm{P}_{3 / 2}$ state). As expected, no drift is observed when the relative spatial phase, $\varphi$, is $0, \pi$ or $2 \pi$. For all other phase shifts, the data of fig. 2 clearly show an induced drift, with opposite extrema around $\pi / 2$ and $3 \pi / 2$. The maximum drift velocity observed here is $\approx 0.8 \mathrm{~mm} / \mathrm{s}$, or $1 / 4$ of an atomic recoil. It has been proven in ref. 24 that the drift velocity in the lattice is constant in time, i.e., independent of the interaction time $\tau$.

As the lattice structures are periodic in all three dimensions and the relative spatial shifts can be adjusted independently along the $x, y$ or $z$ directions, the drift can be induced in any direction in space. This was evidenced in 24] by direct imaging of the atom cloud.

\section{Classical simulations}

In order to understand the qualitative behaviour of our Brownian motor, we have performed simulations of a classical atomic cloud in an optical lattice. We thus consider Brownian particles which can be in one of two internal states, indexed by $j$. The Brownian motion is characterised by the momentum diffusion constant $D_{\mathrm{v}}(\mathbf{x})$ and the external potentials $U_{j}(\mathbf{x})$. The FokkerPlanck equation (FPE) 34 for the probability distribution $W_{j}(\mathbf{x}, \mathbf{v}, t)$ of a particle in state $j$ located at $\mathbf{x}$ with velocity $\mathbf{v}$, written in time units of the friction coefficient and in space units of the typical variation scale of the potentials (so that all variables are dimensionless) is given by [6]

$$
\left[\partial_{t}+v \partial_{\mathbf{x}}\right] W_{j}+\partial_{\mathbf{v}}\left[\mathbf{v}+\nabla \tilde{U}_{j}(\mathbf{x})+D_{\mathbf{v}}(\mathbf{x}) \partial_{\mathbf{v}}\right] W_{j}=\tilde{\gamma}_{j^{\prime} \rightarrow j}(\mathbf{x}) W_{j^{\prime}}-\tilde{\gamma}_{j \rightarrow j^{\prime}}(\mathbf{x}) W_{j} .
$$

The actual potentials created by the optical lattices are slightly more complicated than the simple sine function model given in fig. 1 First, the different irradiances of the lasers used to create each lattice results in distinct potential depths. Moreover, the interaction strength also depends on the magnetic sublevel of the atom, such that the latter will feel variations in 

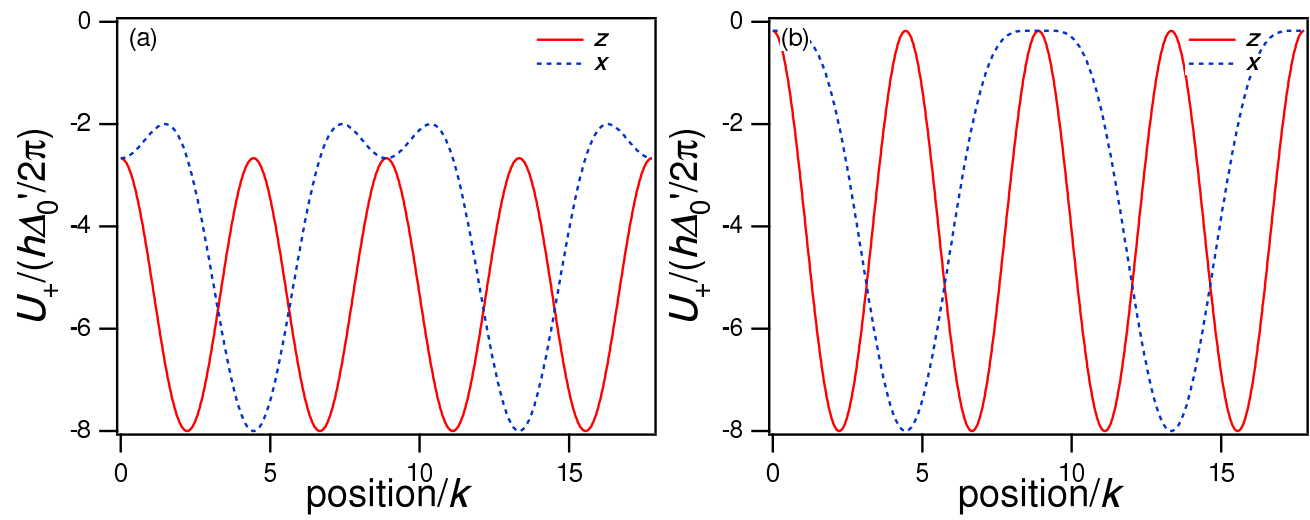

Fig. 3. Optical lattice potential along the $z[U(x=0, y=0, z)]$ and $x[U(x, y=0, z=0)]$ axes for (a) a $J_{\mathrm{g}}=1 / 2 \rightarrow J_{\mathrm{e}}=3 / 2$ transition; (b) an atom in the $F=4, M_{F}=+4$ state, eq. (4).

the potential as it is transferred from one lattice to the other and as it is optically pumped between $M_{F}$ states. Second, the actual potentials in our system have different shapes in different directions.

The simplest atomic system which undergoes a Sisyphus effect consists in two atomic levels of total angular momentum $J_{\mathrm{g}}=1 / 2$ in the ground state and $J_{\mathrm{e}}=3 / 2$ in the excited state 28 . The resulting optical lattice potential is then

$$
U_{ \pm}(\mathbf{x})=\frac{8 \hbar \Delta_{0}^{\prime}}{3}\left[\cos ^{2}\left(k_{x} x\right)+\cos ^{2}\left(k_{y} y\right) \mp \cos \left(k_{x} x\right) \cos \left(k_{y} y\right) \cos \left(k_{z} z\right)\right]
$$

for an atom in the $M_{\mathrm{g}}= \pm 1 / 2$ substate, where $\Delta_{0}^{\prime}$ is the light shift and the $k$ 's are the effective magnitude of the wave vectors along the axes [7. We see that along $z$ the potential is simply sinusoidal, while in the two transverse directions, deep wells are separated by shallower minima, as shown in fig. [3(a).

To get a qualitative picture of the effect of the shape of the potential, we have performed numerical simulations of the FPE (1) in a 2D geometry, using the potential

$$
\tilde{U}_{j}(\mathbf{x})=A_{j}\left[\cos ^{2}\left(x / 2+\varphi_{j, x}\right)+1-\cos \left(x / 2+\varphi_{j, x}\right) \cos \left(z+\varphi_{j, z}\right)\right],
$$

based on eq. (2) for $y=0$, with parameters $A=200, D_{\mathrm{v}}=75$, and $\tilde{\gamma}_{\mathrm{A} \rightarrow \mathrm{B}}=3 \tilde{\gamma}_{\mathrm{B} \rightarrow \mathrm{A}}=7.5$, for a phase shift chosen either in the $z$ or $x$ directions, resulting in a drift velocity $v_{\mathrm{d}_{z}}$ or $v_{\mathrm{d}_{x}}$, respectively. First, we see in fig. 4(a) that the overall variation of the drift velocity as a function of the phase shift along $z$ is very similar to the one observed experimentally (see fig. 2). One main difference is that there is a plateau close to $\varphi=0$ or $2 \pi$ where the drift velocity is very small, i.e., the Brownian motor mechanism is very weak in these regions. In contrast, in the experiment we get a clear directed motion even for minute phase difference between the lattices. Also, the simulation gives a maximum effect for phase shifts of $\varphi \approx 2 \pi / 3$ and $4 \pi / 3$, while in the experiment the maximum drift is seen at $\varphi \approx \pi / 2,3 \pi / 2$.

We observe, as expected, that the shape of the potential has a strong influence on the drift velocity for a given phase shift. A significant Brownian motor effect is seen only when the minima of the deep potentials of both lattices are close together, but slightly displaced. This is easily explained as the atoms spend most of their time close to the absolute minima of the long lived potential B, and the force they feel when switching to the shorter-lived lattice A will be bigger for the steeper slopes of the deep minima than the shallower ones.

In reality, the Brownian motor with caesium atoms will show a less pronounced difference along the different directions, as the hyperfine structure is more complex than that obtained for the $1 / 2 \rightarrow 3 / 2$ atomic transition. Optical pumping from the cooling process leads to a polarisation of the atoms in the lattice, and most will be found in the extreme $M_{F}= \pm F$ 

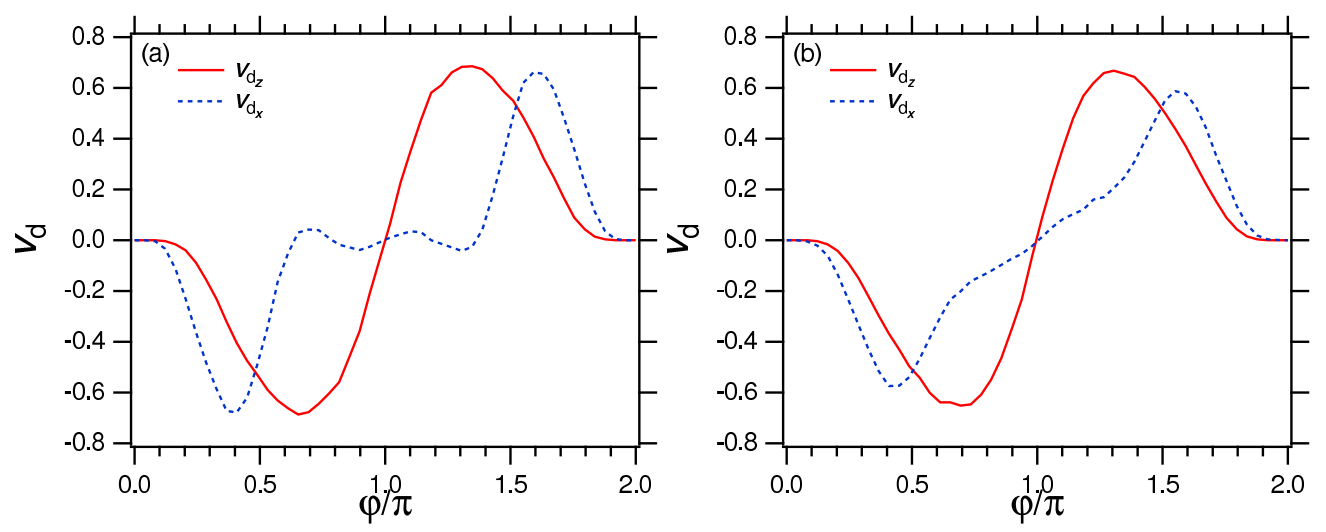

Fig. 4. Results from numerical simulations of the Brownian motor mechanism for the vertical $(z)$ and horizontal $(x)$ directions: (a) for a $J_{\mathrm{g}}=M_{\mathrm{g}}=1 / 2$ atom; (b) for a $F_{\mathrm{g}}=M_{F}=4$ atom. The drift velocity $v_{\mathrm{d}}$ is plotted as a function of the relative spatial phase $\varphi \equiv \varphi_{\mathrm{A}}-\varphi_{\mathrm{B}}$, varied independently along $z$ or $x$.

states. For a caesium atom in the $F_{\mathrm{g}}=4, M_{F}= \pm 4$ state, the lattice potential is given by

$$
U_{ \pm}(\mathbf{x})=\frac{4 \hbar \Delta_{0}^{\prime}}{45}\left\{23\left[\cos ^{2}\left(k_{x} x\right)+\cos ^{2}\left(k_{y} y\right)\right] \mp 44 \cos \left(k_{x} x\right) \cos \left(k_{y} y\right) \cos \left(k_{z} z\right)\right\} .
$$

The potential is still sinusoidal along $z$, while in the two transverse directions, the minima are separated by regions where the potential is essentially flat, as shown in fig. 3(b). Running the same simulations as before, but using now

$$
\tilde{U}_{j}(\mathbf{x})=A_{j}\left\{23 / 44\left[\cos ^{2}\left(x / 2+\varphi_{j, x}\right)+1\right]-\cos \left(x / 2+\varphi_{j, x}\right) \cos \left(z+\varphi_{j, z}\right)\right\},
$$

we see in fig. 4 (b) that the difference between the $x$ and $z$ directions is less pronounced than before.

\section{Towards a quantum Brownian motor}

As seen in the previous section, the main features of our Brownian motor can be qualitatively described using a purely classical model. This is because, although of an essential quantum nature, the coupling between the potentials is driven by spontaneous emission processes which can be treated as semi-classical random jumps 35. Nevertheless, it opens the way for the creation of a quantum Brownian motor [36]. It could be realised at very low temperatures, for instance, at high potential depths, where the motion will clearly be quantised, or by going to a regime where tunnelling between potential wells will become important. Another possibility is to explore possible quantum resonances that are predicted for atomic ratchets 37, 38.

One difficulty with the current setup is that the parameters that are the potential depth, the magnitude of the diffusion, and the transition rates between the two lattices all depend on both the irradiance and the detuning of the optical lattice lasers. Changing either thus results in a different set of parameters. Going to far-detuned lattices and adding additional lasers to induce diffusion and control the transfer between the lattices would allow for an investigation of the effect of individual parameters.

\section{Conclusion}

In summary, we have realised a Brownian motor for cold atoms, based on a dissipative double optical lattice. This is based on a new rectification mechanism of noise in which the potentials 
are all symmetric but spatially shifted with asymmetric transition rates. As our Brownian motor relies on spatially symmetric potentials, it allows for a great control of the direction and magnitude of the induced drift velocity. Numerical simulations have shown that a simple

model describing the motion of Brownian particles shifting between two sinusoidal potentials captures the main features of this Brownian motor. Because of the level of control it offers, our system is promising for the study of the general properties and dynamics of Brownian motors. In particular, it should allow the investigation of quantum versions of these.

\section{Acknowledgements}

This research was conducted using the resources of the High Performance Computing Center North (HPC2N). We thank the Knut och Alice Wallenbergs stiftelse, Carl Tryggers stiftelse, Kempestiftelserna, Magnus Bergwalls stiftelse, the Swedish Research Council, and SIDA/SAREC for financial support.

\section{References}

1. P. Hänggi, F. Marchesoni, and F. Nori, Ann. Phys. (Leipzig) 14, 51 (2005).

2. P. Reimann, Phys. Rep. 361, 57 (2002).

3. P. Curie, Journal de Physique, 3e série 3, 393 (1894).

4. R. P. Feynman, R. B. Leighton, and M. Sands, in The Feynman Lectures on Physics (AddisonWesley, Reading, Mass., 1963), Chap. 46.

5. M. von Smoluchowski, Phys. Z. 13, 1069 (1912).

6. L. Sanchez-Palencia, Phys. Rev. E 70, 011102 (2004).

7. G. Grynberg and C. Robilliard, Phys. Rep. 355, 335 (2001).

8. P. S. Jessen and I. H. Deutsch, Adv. At. Mol. Opt. Phys. 37, 95 (1996).

9. G. Grynberg, C. Triché, L. Guidoni, and P. M. Visser, Europhys. Lett. 51, 506 (2000).

10. P. M. Visser and G. Grynberg, Eur. Phys. J. D 12, 403 (2000).

11. P. Horak, J.-Y. Courtois, and G. Grynberg, Phys. Rev. A 58, 3953 (1998).

12. G. Grynberg, P. Horak, and C. Mennerat-Robilliard, Europhys. Lett. 49, 424 (2000).

13. L. Guidoni, C. Triché, P. Verkerk, and G. Grynberg, Phys. Rev. Lett. 79, 3363 (1997).

14. L. Guidoni, B. Dépret, A. di Stefano, and P. Verkerk, Phys. Rev. A 60, R4233 (1999).

15. L. Sanchez-Palencia, F.-R. Carminati, M. Schiavoni, F. Renzoni, and G. Grynberg, Phys. Rev. Lett. 88, 133903 (2002).

16. M. Schiavoni, F.-R. Carminati, L. Sanchez-Palencia, F. Renzoni, and G. Grynberg, Europhys. Lett. 59, 493 (2002).

17. L. Sanchez-Palencia and G. Grynberg, Phys. Rev. A 68, 023404 (2003).

18. C. Mennerat-Robilliard, D. Lucas, S. Guibal, J. Tabosa, C. Jurczak, J.-Y. Courtois, and G. Grynberg, Phys. Rev. Lett. 82, 851 (1999).

19. C. Robilliard, D. Lucas, and G. Grynberg, Appl. Phys. A 75, 213 (2002).

20. M. Schiavoni, L. Sanchez-Palencia, F. Renzoni, and G. Grynberg, Phys. Rev. Lett. 90, 094101 (2003).

21. P. H. Jones, M. Goonasekera, and F. Renzoni, Phys. Rev. Lett. 93, 073904 (2004).

22. R. Gommers, P. Douglas, S. Bergamini, M. Goonasekera, P. H. Jones, and F. Renzoni, Phys. Rev. Lett. 94, 143001 (2005).

23. R. Gommers, S. Bergamini, and F. Renzoni, Phys. Rev. Lett. 95, 073003 (2005).

24. P. Sjölund, S. J. H. Petra, C. M. Dion, S. Jonsell, M. Nylén, L. Sanchez-Palencia, and A. Kastberg, Phys. Rev. Lett. 96, 190602 (2006).

25. S. Chu, Rev. Mod. Phys. 70, 685 (1998).

26. C. N. Cohen-Tannoudji, Rev. Mod. Phys. 70, 707 (1998).

27. W. D. Phillips, Rev. Mod. Phys. 70, 721 (1998).

28. J. Dalibard and C. Cohen-Tannoudji, J. Opt. Soc. Am. B 6, 2023 (1989).

29. L. Sanchez-Palencia, P. Horak, and G. Grynberg, Eur. Phys. J. D 18, 353 (2002).

30. H. Ellmann, J. Jersblad, and A. Kastberg, Phys. Rev. Lett. 90, 053001 (2003).

31. H. Ellmann, J. Jersblad, and A. Kastberg, Eur. Phys. J. D 22, 355 (2003). 
32. S. J. H. Petra, P. Sjölund, and A. Kastberg, J. Opt. A.: Pure Appl. Opt. 8, 381 (2006).

33. P. Verkerk, D. R. Meacher, A. B. Coates, J.-Y. Courtois, S. Guibal, C. Salomon, and G. Grynberg, Europhys. Lett. 26, 171 (1994).

34. H. Risken, The Fokker-Planck Equation, 2nd ed. (Springer, Berlin, 1989).

35. G. Lindblad, Commun. Math. Phys. 48, 119 (1976).

36. P. Hänggi and G.-L. Ingold, Chaos 15, 026105 (2005).

37. E. Lundh and M. Wallin, Phys. Rev. Lett. 94, 110603 (2005).

38. E. Lundh, Phys. Rev. E 74, 016212 (2006). 